\title{
THE LIFTING TWO-DIMENSIONAL AEROFOIL BETWEEN SLOTTED WALLS
}

\author{
A. H. LOW \\ (received 5 June 1962)
}

\section{Summary}

A mathematical theory is developed which enables the wind-tunnel corrections to the lift and moment forces acting on an aerofoil in subsonic twodimensional flow to be calculated. The usual "averaged" boundary condition for slotted walls is assumed and the corrections obtained by successive approximation from the open-jet tunnel.

\section{Introduction}

Woods [11] has recently revived the problem of determining the blockage interference at an aerofoil symmetrically placed between the slotted walls of a wind-tunnel. Discussions due to Baldwin, Turner and Knechtel [1], Maeder and Wood [5] and others are also available. The results obtained show that the blockage interference may be made to vanish for a particular slot configuration. However, it is still to be expected that the wall interference effects on the lift and moment forces may exist and these should be evaluated if the wind-tunnel measurements are to be corrected in order to predict free air conditions.

These corrections will be considered in this paper. Apparently, little theoretical work has been done on these corrections. The only discussion available to the writer is that of Maeder and Wood [5] who determine the angle of incidence correction in terms of the lift coefficient. However, their work does not attempt the problem of determining the lift and moment corrections as defined in $\S 5$.

Consider the subsonic flow of an inviscid fluid past a thin aerofoil placed at a small incidence $\alpha$ midway between the slotted walls (Figure 1). The incidence is measured relative to the direction of the undisturbed stream, which is assumed parallel to the walls. At the slotted walls, the actual boundary condition pertaining is replaced by an "averaged" boundary condition (see, for example, [11])

$$
\frac{\partial \phi}{\partial x}+A \frac{\partial^{2} \phi}{\partial x \partial n}=0
$$


where $\phi$ is the (perturbation) potential of the flow in the tunnel; $x$ is the direction of the walls; $n$ is the outward normal to the walls and $A$ is a constant related to the slot geometry. The special cases of $A$ being zero and tending to infinity correspond to the open jet and the solid wall tunnel respectively.

The changes in the aerofoil's lift and moment coefficients, due to the imposition on the boundaries of the stream of the condition expressed in equation (1), are considered. A method of calculation based on a known solution of Laplace's equation in a given region and the successive approximations solution to a Fredholm type integral equation, is outlined. This method allows the calculation of the coefficients for given values of three variables. These variables are the incidence $\alpha$; the ratio $c / 2 \beta h$ where $c$ is the aerofoil chord, $2 h$ is the tunnel height and $\beta$ is related to the Mach number of the flow (see $\S 2$ ); and the ratio $A / h$.

\section{The boundary value problem}

Let $(q, \theta)$ be the polar components of the velocity vector of the stream flow. At infinity upstream (i.e. $x=-\infty$ ) we assume this velocity has components $(U, 0)$.

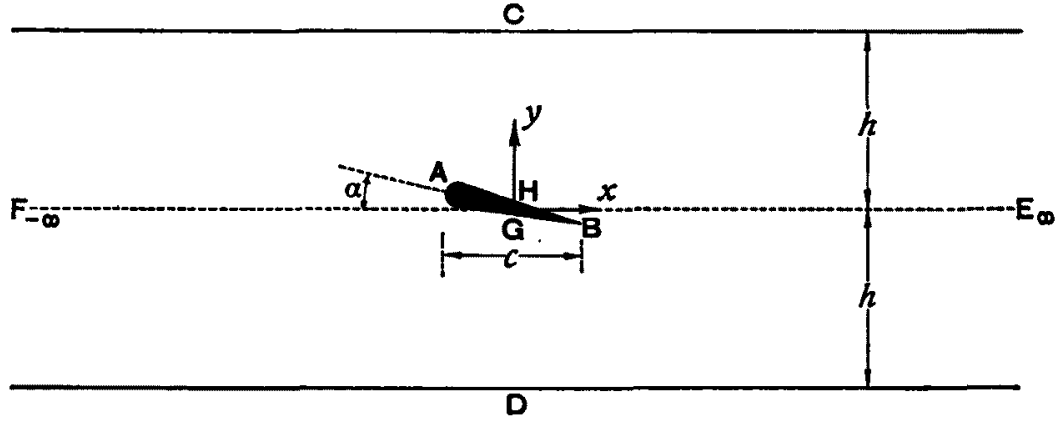

Fig. 1

Consider the complex function $f$ defined by

$$
f=\Omega+i \theta
$$

where

$$
\begin{aligned}
& \Omega=-\beta \int_{0}^{q} \frac{d q}{q} ; \\
& \beta^{2}=1-M^{2}
\end{aligned}
$$

and $M$ is the Mach number of the flow at infinity upstream. For thin aerofoils at small incidences, the ratio $(U-q) / U$ is a small first-order quantity 
everywhere except in the neighbourhoods of the two stagnation points of the aerofoil. Therefore, throughout the strip $-\infty \leqq x \leqq \infty,-h \leqq y \leqq h$ with the exception of the stagnation points,

$$
\Omega \doteqdot \frac{U-q}{U}
$$

and $f$ is an analytic function of $Z$, where

$$
Z=x+i \beta y
$$

(see, for example, Robinson and Laurmann, [6], p. 333). Further, the above assumptions allow the imposition of the flow direction at the aerofoil surface on the interval $-c / 2 \leqq x \leqq c / 2, y=0$. That is.

$$
\theta=\theta(x) \quad-\frac{c}{2} \leqq x \leqq \frac{c}{2}, \quad y=0 .
$$

On the tunnel walls, we have, from equation (1),

$$
\begin{array}{lll}
\Omega+A \frac{\partial \Omega}{\partial y}=0 & -\infty \leqq x \leqq \infty, & y=h, \\
\Omega-A \frac{\partial \Omega}{\partial y}=0 & -\infty \leqq x \leqq \infty, & y=-h .
\end{array}
$$

The $Z$-plane can be mapped into a suitable singly-connected region, namely a rectangle in the $\zeta(=\gamma+i \eta)$-plane (see figure 2$)$, by means of a con iormal transformation involving Jacobian elliptic functions. The transformation (Woods [8]) is

$$
\operatorname{cn}(\zeta ; k)=-\frac{k^{\prime}}{k} \sinh \left(\frac{\pi Z}{2 \beta h}\right)
$$

where the modulii of the elliptic functions, $k$ and $k^{\prime}$, are given by

$$
k=\tanh \frac{\pi c}{4 \beta h} .
$$

In the $\zeta$-plane, the aerofoil surface $-c / 2 \leqq x \leqq c / 2, y=0$ maps into the line $-2 K \leqq \gamma \leqq 2 K, \eta=0$; the upper and lower walls $-\infty \leqq x \leqq \infty$, $y=h$ and $y=-h$ become $0 \leqq \gamma \leqq 2 K, \eta=K^{\prime}$ and $-2 K \leqq \gamma \leqq 0$, $\eta=K^{\prime}$ respectively. Corresponding points in figures (1) and (2) are marked accordingly. The numbers $K$ and $K^{\prime}$ are the real and imaginary quarter periods of the elliptic functions of modulus $k$. Because of the conformal nature of the transformation (9), $f$ will be an analytic function of $\zeta$ within and on the rectangle $-2 K \leqq \gamma \leqq 2 K, 0 \leqq \eta \leqq K^{\prime}$ except at the points 


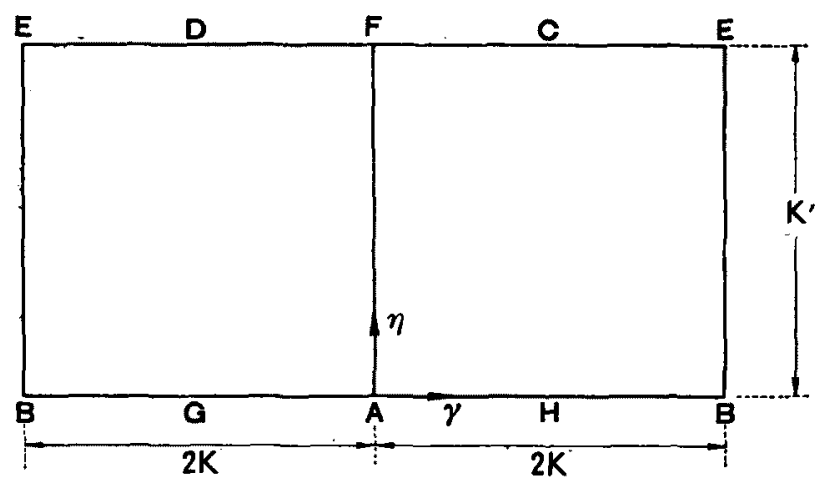

Fig. 2

corresponding to singularities in the $Z$-plane. Such singular points, in the $\zeta$-plane, will thus be confined to the line $-2 K \leqq \gamma \leqq 2 K, \eta=0$.

The form assumed by the boundary conditions (equations (7) and (8)) in the $\zeta$-plane will now be considered. Equation (7), which states that the flow direction on the aerofoil surface is a known function, becomes

$$
\theta=\theta(\gamma) \quad-2 K \leqq \gamma \leqq 2 K, \quad \eta=0 .
$$

By differentiation of (9), using the addition properties of the hyperbolic and elliptic functions,

$$
d Z=\frac{2 \beta h k}{\pi} \operatorname{sn} \zeta d \zeta
$$

But, on the tunnel walls $\zeta=\gamma+i K^{\prime}$, so that the equations (8) become, with the aid of (12),

$$
\begin{aligned}
& \Omega(\gamma)+\frac{\pi A}{2 h} \operatorname{sn} \gamma \frac{\partial \Omega}{\partial \eta}=0 \quad 0 \leqq \gamma \leqq 2 K, \quad \eta=K^{\prime} \\
& \Omega(\gamma)-\frac{\pi A}{2 h} \operatorname{sn} \gamma \frac{\partial \Omega}{\partial \eta}=0 \quad-2 K \leqq \gamma \leqq 0, \quad \eta=K^{\prime} .
\end{aligned}
$$

Moreover, from figures 1 and 2 it is clear that $f$ must satisfy the relation

$$
\lim _{\varepsilon \rightarrow 0} f(-2 K+\varepsilon, \eta)=\lim _{\varepsilon \rightarrow 0} i(2 K-\varepsilon, \eta) \quad \varepsilon>0, \quad 0 \leqq \eta \leqq K^{\prime} .
$$

Therefore, the boundary value problem is to determine an analytic function $f(\zeta)$ which satisfies equations (11), (13) and (14).

\section{The solution of the boundary value problem}

The function $f(\zeta)$ which satisfies equation (14) and the conditions that (i) $\theta$ is known on $-2 K \leqq \gamma \leqq 2 K, \eta=0$ and 
(ii) $\Omega$ is known on $-2 K \leqq \gamma \leqq 2 K, \eta=K^{\prime}$, is given by [8]

(15) $f(\zeta)=\frac{1}{2 \pi} \int_{-2 K}^{2 K}\left\{\theta\left(\gamma^{*}\right) \frac{\mathrm{sn}^{\prime}}{\mathrm{sn}}\left(\frac{\gamma^{*}-\zeta}{2}\right)+i \Omega\left(\gamma^{*}+i K^{\prime}\right) \frac{\mathrm{sn}^{\prime}}{\mathrm{sn}}\left(\frac{\gamma^{*}-\zeta+i K^{\prime}}{2}\right)\right\} d \gamma^{*}$

where $\operatorname{sn}^{\prime} / \operatorname{sn}(u)$ denotes $d / d u(\log \operatorname{sn} u)$. The following identities [9] are useful;

$$
\frac{\mathrm{sn}^{\prime}}{\mathrm{sn}}(u)=\mathrm{ds} 2 u+\operatorname{cs} 2 u
$$

and

(17) $(\operatorname{sn} u-\operatorname{sn} v)(\operatorname{ds}(u-v)+\operatorname{cs}(u-v))=\operatorname{dn} u \operatorname{cn} v+\operatorname{cn} u \operatorname{dn} v$.

The equations (13) may be written

$$
\Omega+\frac{\pi A}{2 h}|\operatorname{sn} \gamma| \frac{\partial \Omega}{\partial \eta}=0 \quad-2 K \leqq \gamma \leqq 2 K, \quad \eta=K^{\prime} .
$$

That is, on using the Cauchy-Riemann relations for an analytic function,

$$
\Omega-\frac{\pi A}{2 h}|\operatorname{sn} \gamma| \frac{\partial \theta}{\partial \gamma}=0 \quad-2 K \leqq \gamma \leqq 2 K, \quad \eta=K^{\prime} .
$$

Therefore, from equations (15), (16) and (18), we find on integrating by parts

$$
\begin{aligned}
f(\zeta) & =\frac{1}{2 \pi} \int_{-2 K}^{2 K}\left\{\theta\left(\gamma^{*}\right)\left(\mathrm{ds}\left(\gamma^{*}-\zeta\right)+\operatorname{cs}\left(\gamma^{*}-\zeta\right)\right)\right. \\
& \left.+\frac{\pi A}{2 h} \theta\left(\gamma^{*}+i K^{\prime}\right) \frac{\partial}{\partial \gamma^{*}}\left[\left|\operatorname{sn} \gamma^{*}\right|\left(\operatorname{dn}\left(\gamma^{*}-\zeta\right)+k \operatorname{cn}\left(\gamma^{*}-\zeta\right)\right)\right]\right\} d \gamma^{*} .
\end{aligned}
$$

This last equation gives, providing $\theta\left(\gamma+i K^{\prime}\right)$ is known, the function $f(\zeta)$. Unfortunately, $\theta\left(\gamma+i K^{\prime}\right)$ is not known explicitly. However, on substituting $\zeta=\gamma+i K^{\prime}$ in (19), it is possible to obtain a Fredholm type integral equation for this function, namely,

$$
\begin{aligned}
& \theta\left(\gamma+i K^{\prime}\right)=\frac{1}{2 \pi} \int_{-2 K}^{2 K}\left\{\theta\left(\gamma^{*}\right)\left(\operatorname{dn}\left(\gamma^{*}-\gamma\right)+k \operatorname{cn}\left(\gamma^{*}-\gamma\right)\right)\right. \\
& \left.+\frac{\pi A}{2 h} \theta\left(\gamma^{*}+i K^{\prime}\right) \frac{\partial}{\partial \gamma^{*}}\left[\left|\operatorname{sn} \gamma^{*}\right|\left(\operatorname{ds}\left(\gamma^{*}-\gamma\right)+\operatorname{cs}\left(\gamma^{*}-\gamma\right)\right)\right]\right\} \mathrm{d} \gamma^{*} .
\end{aligned}
$$

Thus, equations (20) and (19) may be regarded as successive steps in the determination of the function $f(\xi)$. The first step, then, is the solution of (20) for given $\theta(\gamma)$. This solution may be obtained by the method of successive approximations. It can be shown that [3] these approximations will 
converge for all values of $A / h$ in the range

$$
\frac{A}{h} \leqq k\left\{\frac{3}{\left(k^{2}\left(K^{2}-E^{2}\right)-(K-E)^{2}\right)}\right\}^{\frac{1}{2}},
$$

where $E$ is the complete elliptic integral of tfie second kind.

\section{The flow direction on the aerofoil surface}

Ignoring the aerofoil thickness and assuming Joukowski's condition that the rear stagnation point remains fixed in position at the trailing edge, the direction of flow on the aerofoil surface is given by [4]

$$
\theta(\gamma)=-\alpha+\pi \lambda \delta(\gamma)
$$

where $\alpha$ is the angle of incidence of the aerofoil (measured from the negative $x$-axis), $\lambda$ is a number initially unknown and due to the small movement of the front stagnation point away from its mean position $\gamma=0$ and $\delta(\gamma)$ is the delta function.

The value of $\lambda$ is fixed by the condition that the incidence of the aerofoil has no effect on the flow direction at infinity upstream. It is assumed in $\$ \mathbf{2}$ that $\theta=0$ at $x=-\infty$. But, in the $\zeta$-plane, the point $x=-\infty$ maps into the point $\zeta=i K^{\prime}$. Therefore,

$$
\theta\left(i K^{\prime}\right)=0 \text {. }
$$

Equation (23) fixes the value of $\lambda$ in terms of $\alpha$.

\section{The lift and moment coefficients}

The lift coefficient, $C_{L}$, and the moment coefficient about the chord midpoint, $C_{M}$, are given by $[10]$

$$
C_{L}=-\frac{4 h k}{\pi c} \int_{-2 K}^{2 X} \Omega(\gamma) \operatorname{sn} \gamma d \gamma
$$

and

$$
C_{M}=8 \beta k\left(\frac{h}{\pi c}\right)^{2} \int_{-2 K}^{2 K} \Omega(\gamma) \operatorname{sn} \gamma \log \left|\frac{\operatorname{dn} \gamma-k \operatorname{cn} \gamma}{k^{\prime}}\right| d \gamma
$$

respectively. The value of $\Omega(\gamma)$ to be inserted in these expressions is the right-hand side of (19) with $\zeta=\gamma$. This function, in turn, depends on the value $\theta\left(\gamma+i K^{\prime}\right)$ obtained from equation $(20)$.

The first approximation to $\theta\left(\gamma+i K^{\prime}\right), \theta_{1}\left(\gamma+i K^{\prime}\right)$ say, is, from $(20)$,

$$
\theta_{1}\left(\gamma+i K^{\prime}\right)=\frac{1}{2 \pi} \int_{-2 K}^{2 K} \theta\left(\gamma^{*}\right)\left(\operatorname{dn}\left(\gamma^{*}-\gamma\right)+k \operatorname{cn}\left(\gamma^{*}-\gamma\right)\right) d \gamma
$$


Using equation (22) this becomes

$$
\theta_{1}\left(\gamma+i K^{\prime}\right)=-\frac{\lambda}{2}(\operatorname{dn} \gamma+k \operatorname{cn} \gamma)-\alpha .
$$

Substitution of (22) and (26) for $\theta(\gamma)$ and $\theta\left(\gamma+i K^{\prime}\right)$ respectively in the right-hand side of $(20)$ gives the second approximation to $\theta\left(\gamma+i K^{\prime}\right)$, $\theta_{2}\left(\gamma+i K^{\prime}\right)$ say. This is,

$$
\begin{aligned}
& \theta_{2}\left(\gamma+i K^{\prime}\right)=-\left(\frac{\lambda}{2}(\operatorname{dn} \gamma+k \operatorname{cn} \gamma)+\alpha\right) \\
&-\frac{A}{4 h} \int_{-2 K}^{2 K}\left(\frac{\lambda}{2}\left(\operatorname{dn} \gamma^{*}+k \operatorname{cn} \gamma^{*}\right)+\alpha\right) \\
& \frac{\partial}{\partial \gamma^{*}}\left(\left|\operatorname{sn} \gamma^{*}\right|\left[\operatorname{ds}\left(\gamma^{*}-\gamma\right\rangle+\operatorname{cs}\left(\gamma^{*}-\gamma\right)\right]\right) d \gamma^{*}
\end{aligned}
$$

Substitution of (22) and (27) for $\theta(\gamma)$ and $\theta\left(\gamma+i K^{\prime}\right)$, respectively, in the right-hand side of $(20)$ gives the third approximation to $\theta\left(\gamma+i K^{\prime}\right)$ and so on.

From equation (19), using (22) and (27), we have to second order in $A / h$,

$$
\begin{aligned}
& \Omega(\gamma)=-\frac{\lambda}{2}\{(\mathrm{ds} \gamma+\operatorname{cs} \gamma) \\
&+\frac{A}{4 h} \int_{-2 K}^{2 K}\left(\operatorname{dn} \gamma^{*}+k \operatorname{cn} \gamma^{*}\right) \\
& \frac{\partial}{\partial \gamma^{*}}\left(\left|\operatorname{sn} \gamma^{*}\right|\left[\mathrm{dn}\left(\gamma^{*}-\gamma\right)+k \operatorname{cn}\left(\gamma^{*}-\gamma\right)\right]\right) \mathrm{d} \gamma^{*} \\
&\left.=\frac{\partial}{4 h}\right)^{2} \int_{-2 K}^{2 K}\left(\left|\operatorname{sn} \gamma^{\prime}\right|\left[\mathrm{ds}\left(\gamma^{\prime}-\gamma^{*}\right)+\operatorname{cs}\left(\gamma^{\prime}-\gamma^{*}\right)\right]\right) \delta \\
&\left.\frac{\partial}{\partial \gamma^{\prime}}\left(\left|\operatorname{sn} \gamma^{*}\right|\left[\mathrm{dn}\left(\gamma^{*}-\gamma\right)+k \operatorname{cn}\left(\gamma^{*}-\gamma\right)\right]\right) \mathrm{d} \gamma^{\prime} \mathrm{d} \gamma^{*}\right\} .
\end{aligned}
$$

Using the results obtained in the appendix together with the results that [2]

$$
\begin{gathered}
\int_{0}^{K} \operatorname{sn} \gamma d \gamma=\frac{1}{k} \log \frac{k^{\prime}}{1-k} ; \\
\int_{0}^{K} \operatorname{sn}^{3} \gamma d \gamma=\frac{1}{2 k^{3}}\left(\left(1+k^{2}\right) \log \frac{k^{\prime}}{1-k}-k\right) ;
\end{gathered}
$$

and 


$$
\int_{0}^{K} \operatorname{sn}^{5} \gamma d \gamma=\frac{1}{2 k^{2}}\left(3\left(1+k^{2}\right) \int_{0}^{K} \operatorname{sn}^{3} \gamma d \gamma-2 \int_{0}^{K} \operatorname{sn} \gamma d \gamma\right),
$$

we find, from (24) and (28), after some elementary integration

$$
\begin{aligned}
C_{L}=\frac{4 h k \lambda}{c} & \left\{1+\frac{1}{2 k}\left(\frac{A}{h}\right)\left(k-\left(1-k^{2}\right) \log \frac{k^{\prime}}{1-k}\right)\right. \\
& \left.-\frac{1}{8 k}\left(\frac{A}{h}\right)^{2}\left(k-2 k^{\prime 2} \log \frac{k^{\prime}}{1-k}+\frac{k^{\prime 2}\left(1+3 k^{2}\right)}{k}\left[\log \frac{k^{\prime}}{1-k}\right]^{2}\right)\right\} .
\end{aligned}
$$

Similarly, from (25) and (28), we have

$$
\begin{array}{r}
C_{M}=-\frac{4 \beta \lambda}{\pi}\left(\frac{h}{c}\right)^{2}\left\{\log \left(1-k^{2}\right)+\frac{1}{2 k}\left(\frac{A}{h}\right)\left(\left(1-k^{2}\right) \log \left(1-k^{2}\right) \log \frac{k^{\prime}}{1-k}\right.\right. \\
\left.+3 k \log \left(1-k^{2}\right)+4 k^{2} \log \frac{k^{\prime}}{1-k}\right) \\
+\frac{1}{8 k^{2}}\left(\frac{A}{h}\right)^{2}\left(k^{\prime 2}\left[\log \frac{k^{\prime}}{1-k}\right]^{2}\left[\left(3+k^{2}\right) \log k^{\prime}-4 k^{2}\right]\right. \\
\left.\left.-2 k \log k^{\prime} \log \frac{k^{\prime}}{1-k}\left(7-k^{2}\right)+3 k^{2} \log k^{\prime}\right)\right\} .
\end{array}
$$

Equation (23) gives, using (27) and the result in part (i) of the appendix,

$$
2 \alpha=\lambda(1+k)\left\{1+\frac{A}{2 h k}\left((1-k)^{2} \log \frac{k^{\prime}}{1-k}-k\right)\right\} .
$$

Elimination of $\lambda$ from equations (29), (30) and (31) gives the lift and moment coefficients experienced by an aerofoil at incidence $\alpha$ in a slotted windtunnel.

\section{The wind-tunnel corrections}

For practical purposes it is convenient to eliminate $k$ in favour of the ratio $c / \delta h$ as this ratio is generally kept as small as possible. To do this we use the following expansions applicable for small values of $k$;

$$
\log k^{\prime}=-\frac{k^{2}}{2}-\frac{k^{4}}{4}+O\left(k^{8}\right)
$$

and

$$
\log \frac{k^{\prime}}{1-k}=k+\frac{k^{3}}{3}+O\left(k^{5}\right)
$$

Moreover, for small values of $k$, we have [2] 


$$
K=\frac{\pi}{2}\left(1+\frac{k^{2}}{4}\right)+O\left(k^{4}\right)
$$

and

$$
E=\frac{\pi}{2}\left(1-\frac{k^{2}}{4}\right)+O\left(k^{4}\right) .
$$

Therefore, from (21), the successive approximations solution will converge for $A / h \leqq 4 / \pi k$.

Equations (31), (32) and (33) give, to second order in $k$ and $A / h$,

$$
\lambda=2 \alpha\left\{1-k\left(1-\frac{A}{h}\right)+k^{2}\left(1-\frac{5}{3} \frac{A}{h}+\left(\frac{A}{h}\right)^{2}\right)\right\} .
$$

Also, from (29), (32) and (33) we find

$$
C_{L}=\frac{4 h k \lambda}{c}\left\{1+\frac{k^{2}}{3} \frac{A}{h}-\frac{k^{2}}{2}\left(\frac{A}{h}\right)^{2}\right\}
$$

and

$$
C_{M}=\frac{4 \beta \lambda}{\pi}\left(\frac{h}{c}\right)^{2} k^{2}\left\{\left(1+\frac{k^{2}}{2}\right)-\frac{7 k^{2}}{12}\left(\frac{A}{h}\right)^{2}\right\} .
$$

From (10) we have, to third order in $c / \delta h$,

$$
k=\frac{1}{4} \frac{\pi c}{\beta h}\left\{1-\frac{1}{48}\left(\frac{\pi c}{\beta h}\right)^{2}\right\} .
$$

Therefore, from (34), (35), (36) and (37) we have, in terms of $c / \delta h$,

$$
C_{L}=\frac{2 \pi \alpha}{\beta}\left\{1-\frac{\pi c}{4 \beta h}\left(1-\frac{A}{h}\right)+\frac{1}{86}\left(\frac{\pi c}{\beta h}\right)^{2}\left(4-8 \frac{A}{h}+3\left(\frac{A}{h}\right)^{2}\right)\right\}
$$

and

(39) $\quad C_{M}=\frac{\pi \alpha}{2 \beta}\left\{1-\frac{\pi c}{4 \beta h}\left(1-\frac{A}{h}\right)+\frac{5}{182}\left(\frac{\pi c}{\beta h}\right)^{2}\left(2-4 \frac{A}{h}+\left(\frac{A}{h}\right)^{2}\right)\right\}$.

In an infinite stream $h \rightarrow \infty$ so that (38) and (39) yield

and

$$
C_{L_{\infty}}=\frac{2 \pi \alpha}{\beta}
$$

$$
C_{M_{\infty}}=\frac{\pi \alpha}{2 \beta}
$$

the classical results for an infinite (free) stream. Therefore, from (38) and (39) 


$$
\frac{C_{L}}{C_{L_{\infty}}}=1-\frac{1}{2}\left(\frac{\pi c}{2 \beta h}\right)\left(1-\frac{A}{h}\right)+\frac{1}{24}\left(\frac{\pi c}{2 \beta h}\right)^{2}\left(4-8 \frac{A}{h}+3\left(\frac{A}{h}\right)^{2}\right)
$$

and

$$
\frac{C_{M}}{C_{M_{\infty}}}=1-\frac{1}{2}\left(\frac{\pi c}{2 \beta h}\right)\left(1-\frac{A}{h}\right)+\frac{5}{48}\left(\frac{\pi c}{2 \beta h}\right)^{2}\left(2-4 \frac{A}{h}+\left(\frac{A}{h}\right)^{2}\right)
$$

respectively. These last two equations give the corrections to be applied to slotted wind-tunnel measurements of the lift and moment coefficients in order to predict free air conditions.

In particular, it is known that a value of $A / h \doteqdot 1.2$ is required for zero blockage in a slotted tunnel (see, for example, [1]). Substituting this value of $A / h$ in (40) and (41) we find

and

$$
\frac{C_{L}}{C_{L_{\infty}}}=1+\frac{1}{10}\left(\frac{\pi c}{2 \beta h}\right)-\frac{4}{75}\left(\frac{\pi c}{2 \beta h}\right)^{2}
$$

$$
\frac{C_{M}}{C_{M_{\infty}}}=1+\frac{1}{10}\left(\frac{\pi c}{2 \beta h}\right)-\frac{17}{120}\left(\frac{\pi c}{2 \beta h}\right)^{2}
$$

If we choose, for illustrative purposes, $c / 2 \beta h=1 / 10$ these last two equations give for the lift and moment corrections

and

$$
C_{L}=1.0262 C_{L_{\infty}}
$$

$$
C_{M}=1.0176 C_{M_{\infty}} \text {. }
$$

\section{Appendix}

In the determination of equations (29), (30) and (31) from equations (24), (25), (27) and (28) we require, amongst others, the following integrals;

(i)

$$
\begin{array}{r}
I_{1}(v)=\int_{-2 K}^{2 K}(\operatorname{dn} u+k \operatorname{cn} u) \frac{\partial}{\partial u}(|\operatorname{sn} u|[\operatorname{dn}(u-v)+k \operatorname{cn}(u-v)]) d u \\
=\int_{0}^{2 K}(\operatorname{dn} u+k \operatorname{cn} u) \frac{\partial}{\partial u}(|\operatorname{sn} u|[\operatorname{dn}(u-v)-\operatorname{dn}(u+v) \\
+k \operatorname{cn}(u-v)-k \operatorname{cn}(u+v)]) d u .
\end{array}
$$

That is, on integrating by parts and using the addition theorems for the elliptic functions,

$$
I_{1}=2 k^{2} \operatorname{sn} v \int_{0}^{2 K} \operatorname{sn}^{3} u(\operatorname{dn} u+k \operatorname{cn} u) \frac{\operatorname{dn} u \operatorname{dn} v+k \operatorname{cn} u \operatorname{cn} v}{1-k^{2} \operatorname{sn}^{2} u \operatorname{sn}^{2} v} d u .
$$

As cn $u$ is an odd function about $u=K$ we find 


$$
I_{1}=4 k^{2} \operatorname{sn} v \int_{0}^{K} \operatorname{sn}^{3} u \frac{\left(\mathrm{dn} v+k^{2} \operatorname{cn} v\right)-k^{2} \operatorname{sn}^{2} u(\operatorname{dn} v+\operatorname{cn} v)}{1-k^{2} \operatorname{sn}^{2} u \operatorname{sn}^{2} v} d u
$$

which, after some algebra, becomes

$$
\begin{array}{r}
I_{1}=\frac{4}{\operatorname{sn} v}\left\{k^{2}(\operatorname{dn} v+\operatorname{cn} v) \int_{0}^{K} \operatorname{sn}^{3} u d u+\frac{\operatorname{cn} v \operatorname{dn} v}{\operatorname{sn}^{2} v}(\operatorname{cn} v+\operatorname{dn} v) \int_{0}^{K} \operatorname{sn} u d u\right. \\
\left.-\frac{\operatorname{cn} v \operatorname{dn} v(\operatorname{cn} v+\operatorname{dn} v)}{\operatorname{sn}^{2} v} \int_{0}^{K} \frac{\operatorname{sn} u d u}{1-k^{2} \operatorname{sn}^{2} u \operatorname{sn}^{2} v}\right\} .
\end{array}
$$

Using the results [2]

$$
\begin{aligned}
\int_{0}^{K} \frac{\operatorname{sn} u d u}{1-\alpha^{2} \operatorname{sn}^{2} u}= & \frac{1}{\sqrt{\left(1-\alpha^{2}\right)\left(k^{2}-\alpha^{2}\right)}} \log \left|\frac{k^{\prime}}{\sqrt{1-\alpha^{2}}-\sqrt{k^{2}-\alpha^{2}}}\right| ; \\
& \int_{0}^{K} \operatorname{sn} u d u=\frac{1}{k} \log \frac{k^{\prime}}{1-k} ;
\end{aligned}
$$

and

$$
\int_{0}^{K} \operatorname{sn}^{3} u d u=\frac{1}{2 k^{3}}\left\{\left(1+k^{2}\right) \log \frac{k^{\prime}}{1-k}-k\right\}
$$

we find that

$$
\begin{aligned}
I_{1}=2 \frac{\operatorname{cn} v+\operatorname{dn} v}{k \operatorname{sn} v}\left\{\left(1+k^{2}\right) \log \frac{k^{\prime}}{1-k}\right. & -k+2 \frac{\operatorname{cn} v \operatorname{dn} v}{\operatorname{sn}^{2} v} \log \frac{k^{\prime}}{1-k} \\
& \left.-\frac{2}{\operatorname{sn}^{2} v} \log \left|\frac{k^{\prime}}{\operatorname{dn} v-k \operatorname{cn} v}\right|\right\} .
\end{aligned}
$$

A useful result may be obtained by letting $v=\gamma+i K^{\prime}$. This is, $\int_{-2 K}^{2 K}(\operatorname{dn} u+k \operatorname{cn} u) \frac{\partial}{\partial u}(|\operatorname{sn} u|[\operatorname{cs}(u-\gamma)+\operatorname{ds}(u-\gamma)]) d u$

$$
\begin{array}{r}
=-\frac{2}{k}(\operatorname{dn} \gamma+k \operatorname{cn} \gamma)\left\{\left(1+k^{2}\right) \log \frac{k^{\prime}}{1-k}-k-2 k \operatorname{dn} \gamma \operatorname{cn} \gamma \log \frac{k^{\prime}}{1-k}\right. \\
\left.-2 k^{2} \operatorname{sn}^{2} \gamma \log \left|\frac{k^{\prime} \operatorname{sn} \gamma}{\operatorname{cn} \gamma-\operatorname{dn} \gamma}\right|\right\} .
\end{array}
$$

(ii) $\quad \int_{-2 K}^{2 K} \operatorname{sn} u\left(\operatorname{dn}\left(u^{\prime}-u\right)+k \operatorname{cn}\left(u^{\prime}-u\right)\right) d u$

$$
=i \int_{-2 K}^{2 K} \operatorname{sn} u(\operatorname{cs}(v-u)+\mathrm{ds}(v-u)) d u
$$

where $v=u^{\prime}+i K^{\prime}$. Using equation (17) we write

$$
\begin{array}{r}
\int_{-2 K}^{2 K} \operatorname{sn} u(\operatorname{cs}(v-u)+\operatorname{ds}(v-u)) d u=\operatorname{sn} v \\
\int_{-2 K}^{2 K}(\operatorname{cs}(v-u)+\operatorname{ds}(v-u)) d u \\
-\int_{-2 K}^{2 K}(\operatorname{cn} v \operatorname{dn} u+\operatorname{cn} u \operatorname{dn} v) d u .
\end{array}
$$


Thus, from (16),

$$
\begin{aligned}
\int_{-2 K}^{2 K} \operatorname{sn} u(\operatorname{cs}(v-u)+\mathrm{ds}(v-u)) d u & =-2\left(\operatorname{sn} v\left[\log \operatorname{sn} \frac{v-u}{2}\right]_{-2 R}^{2 K}+\pi \operatorname{cn} v\right) \\
& =2 \pi i(\operatorname{sn} v-i \operatorname{cn} v) .
\end{aligned}
$$

Therefore, on substituting $v=u^{\prime}+i K^{\prime}$ in this last result, we have

(iii)

$$
\begin{gathered}
\int_{-2 K}^{2 K} \operatorname{sn} u\left(\operatorname{dn}\left(u^{\prime}-u\right)+k \operatorname{cn}\left(u^{\prime}-u\right)\right) d u=\frac{2\left(1-\operatorname{dn} u^{\prime}\right)}{k \operatorname{sn} u^{\prime}} . \\
\text { (iii) } \int_{-2 K}^{2 K} \operatorname{sn} u\left(\operatorname{dn}\left(u^{\prime}-u\right)+k \operatorname{cn}\left(u^{\prime}-u\right)\right) \log \left|\frac{k^{\prime}}{\operatorname{dn} u-k \operatorname{cn} u}\right| d u \\
=i \int_{-2 K}^{2 K} \operatorname{sn} u(\operatorname{cs}(v-u)+\operatorname{ds}(v-u)) \log \left|\frac{k^{\prime}}{\operatorname{dn} u-k \operatorname{cn} u}\right| d u \\
=I_{2}, \text { say, }
\end{gathered}
$$

where $v=u^{\prime}+i K^{\prime}$. From (17), we have

$$
I_{2}=i\left(\operatorname{sn} v \cdot J-\int_{-2 K}^{2 K}(\operatorname{cn} v \operatorname{dn} u+\operatorname{cn} u \operatorname{dn} v) \log \left|\frac{k^{\prime}}{\operatorname{dn} u-k \operatorname{cn} u}\right| d u\right)
$$

where

$$
J=\int_{-2 K}^{2 K}(\operatorname{cs}(v-u)+\mathrm{ds}(v-u)) \log \left|\frac{k^{\prime}}{\operatorname{dn} u-k \operatorname{cn} u}\right| d u .
$$

Using (16) we may write

$$
\begin{aligned}
J & =-2 \int_{-2 K}^{2 K} \frac{\partial}{\partial u}\left(\log \mathrm{sn} \frac{v-u}{2}\right) \log \left|\frac{k^{\prime}}{\operatorname{dn} u-k \operatorname{cn} u}\right| d u \\
& =-2 \pi i \log \frac{k^{\prime}}{1-k}-2 k \int_{-2 K}^{2 K} \operatorname{sn} u \log \mathrm{sn} \frac{v-u}{2} d u
\end{aligned}
$$

on integrating by parts. That is,

$$
\begin{aligned}
\frac{\partial J}{\partial v} & =-k \int_{-2 K}^{2 K} \operatorname{sn} u(\operatorname{cs}(v-u)+\mathrm{ds}(v-u)) d u \\
& =-2 \pi k(\operatorname{cn} v+i \operatorname{sn} v)
\end{aligned}
$$

on using the result in part (ii) above. Consequently,

$$
J=2 \pi\left(\sin ^{-1}(k \operatorname{sn} v)+i \log \frac{\mathrm{dn} v-k \operatorname{cn} v}{1-k}\right)
$$

as, when $v=0, J$ has an odd function for its integrand and vanishes. But,

$$
\sin ^{-1} w=-i \log \left(i w+\sqrt{1-w^{2}}\right),
$$


so that, we have

$$
J=-2 \pi i \log \frac{(1-k)(\operatorname{dn} v+i k \operatorname{sn} v)}{\operatorname{dn} v-k \operatorname{cn} v} .
$$

Using the results [8] that

$$
\int_{-2 K}^{2 K} \operatorname{cn} u \log \left|\frac{\operatorname{dn} u-k \operatorname{cn} u}{k^{\prime}}\right| d u=\frac{\pi}{k} \log \left(1-k^{2}\right)
$$

and

$$
\int_{-2 K}^{2 K} \operatorname{dn} u \log \left|\frac{\operatorname{dn} u-k \operatorname{cn} u}{k^{\prime}}\right| d u=0
$$

we, then, have

$$
I_{2}=\frac{2 \pi}{k \operatorname{sn} u^{\prime}}\left(\log \frac{k^{\prime}\left(1-\operatorname{cn} u^{\prime}\right)}{\operatorname{dn} u^{\prime}-\operatorname{cn} u^{\prime}}+\operatorname{cn} u^{\prime} \log k^{\prime}-\log \frac{k^{\prime}}{1-k}\right) .
$$

\section{References}

[1] Baldwin, B. S., Turner, J. B. and Knechtel, E. D., N.A.C.A. Washington, Tech. Note 3176. (1954).

[2] Byrd, P. F. and Friedmann, M. D., Handbook of Elliptic Integrals (Springer-Verlag. Berlin, 1964).

[3] Low, A. H., Unpublished $\mathrm{Ph}$. D. Thesis, The University of New South Wales (1960).

[4] Low, A. H. and Woods, L. C., J. Austr. Math. Soc., 1 (1960) 220.

[6] Maeder, P. F. and Wood, A. D., Z.A.M.P. 7 (1956), 177.

[6] Robinson, A. and Laurmann, J. A., Wing Theory (C.U.P., 1956).

[7] Woods, L. C., Quart. J. Mech. and App. Maths. 7 (1954), 263.

[8] Woods, L. C., Proc. Roy. Soc. A, 229 (1955), 63.

[9] Woods, L. C., Proc. Roy. Soc. A, 229 (1955), 235.

[10] Woods, L. C., Proc. Roy. Soc. A, 242 (1957), 341.

[11] Woods, L. C., Subsonic Plane Flow (C.U.P., 1961).

School of Mathematics,

The University of New South Wales, Kensington, N.S.W. 